Published online 2017 April 13.

Abstract

\title{
A Comparative Study Between HRCT Findings of Invasive Pulmonary Aspergillosis with Serum and Bronchoalveolar Lavage Galactomannan and Fungal Culture in Lung-Heart Transplant Recipients
}

\author{
Homa Khayatzadeh, ${ }^{1,}$ Payam Mehrian, ${ }^{2}$ and Payam Tabarsi \\ ${ }^{1}$ Radiology Resident of Shahid Beheshti University of Medical Science, Tehran, Iran \\ ${ }^{2}$ Masih e Daneshvari Hospital, Chronic Respiratory Diseases Research Center \\ "Corresponding author: Homa Khayatzadeh. E-mail: Archi363@yahoo.com
}

Received 2016 December 21; Accepted 2017 February 08.

\begin{abstract}
Background: Due to the impact of threats on the clinical outcome and survival of the lung-heart transplant recipients, such as invasive pulmonary Aspergillosis, we aimed to compare the high resolution computed tomography (HRCT) scan findings with the serum BAL galactomannan and fungal culture in these patients.

Methods: We reviewed the data of lung-heart transplant recipients within a 5 year period at our center. The inclusion criterion was confirmed invasive pulmonary Aspergillosis. Demographic, clinical, radiologic and laboratory characteristics of the patients were retrieved from their files and reported.

Results: We reviewed the data of 30 patients (mean age $=36.1 \pm 13.3$; Male gender $=22(73.3 \%$ ). Seventeen patients $(56.7 \%)$ patients had double lung transplant, 10 933.3\% had single lung transplant and $3(10 \%)$ had heart-lung transplant. Consolidation was the most observed finding in the HRCT $(n=25)([83.3 \%])$ and it was more present in the left lower lobe of the lung. Ground glass opacity was observed in 19 (63.3\%) of the patients and mostly in the left lower lobe. Pleural thickening and pleural effusion were present in 7 (23.3) and 19 (63.3\%) of the patients, respectively. Small-size nodular infiltration $(<5 \mathrm{~mm})$ was present in 19 (63.3\%) while large-size nodular infiltration (> $5 \mathrm{~mm}$ ) was observed in only $5(16.7 \%)$ patients. Twenty-seven (90\%) patients had positive culture results, while serum and bronchoalveolar lavage galactomannan was positive in 5 (16.7) and 9 (30\%) patients, respectively. Four (13.3\%) patients died during the study period. However, there was no association between the HRCT and laboratory characteristics of the study population.
\end{abstract}

Conclusions: HRCT is helpful in the diagnosis of invasive pulmonary aspergillosis in patients with lung-heart transplant.

This is an abstract presented in the 33rd Iranian congress of radiology (ICR) and the 15th congress of Iranian radiographic science association (IRSA). 